The expression of inhibitory KIRs was significantly lower in SLE patients $(2.83 \pm 2.8 \%)$ compared to controls $(5.54 \pm 2.01 \%) \quad(P=0.001)$ While, stimulatory KIRs were significantly higher in SLE patients $(3.2 \pm 3.7 \%)$ than controls $(1.02 \pm 0.5 \%),(P=0.001)$. Active SLE patients showed significantly increased expression of stimulatory KIRs $(5.29 \pm 4.29 \%)$ than inactive patients $(1.13 \pm 0.89 \%)$, $(P=0.004)$. However, inhibitory KIRs were significantly decreased in active $(1.28 \pm 1.32 \%)$ than inactive SLE patients $(4.38 \pm 3.05 \%),(p=0.003)$.

Expression of stimulatory KIRs correlated positively with ESR $(r=0.3, P=0.04)$ and negatively with $\mathrm{C} 4(r=-0.4, P=0.01)$. In contrast, inhibitory KIRs correlated negatively with $\mathrm{ESR}(r=-0.5, P=0.003)$ and positively with $\mathrm{C} 4(r=0.4, P=0.02)$. Using Receiver operating characteristic (ROC) curve analysis, expression of inhibitory KIRs on NKT-cells predicted disease activity at a cut-off value of $\leq 1.7 \%$ with $80 \%$ sensitivity and $80 \%$ specificity $(P=0.001)$. While, expression of stimulatory KIRs on NKT-cells predicted active disease at a cut-off value of $>1.4 \%$, sensitivity $(85 \%)$, and specificity $(80 \%)(P=0.001)$.

Conclusions: SLE activity is associated with an increased expression of stimulatory KIRs as well as a decreased expression of inhibitory KIRs on NKT cells. This may play a role in the pathogenesis of flares and acceleration of disease activity in SLE and could be a therapeutic target for SLE patients. Disclosure of Interest: None declared

DOI: 10.1136/annrheumdis-2017-eular.3920

\section{SAT0305 HISTOLOGY OF MINOR SALIVARY GLANDS IN PATIENTS WITH SJÖGREN'S SYNDROME, ASSOCIATION WITH CLINICAL AND LABORATORY ASPECTS}

S. Colafrancesco ${ }^{1}$, F. Arienzo ${ }^{1}$, B. Cerbelli ${ }^{2}$, A. Gattamelata ${ }^{1}$, A. Minniti ${ }^{1}$, G. Picarelli ${ }^{1}$, C. Giordano ${ }^{2}$, G. D'Amati ${ }^{2}$, R. Priori ${ }^{1}$, G. Valesini ${ }^{1} .{ }^{1}$ Dipartimento di medicina interna e specialità mediche; ${ }^{2}$ Dipartimento di radiologia, Oncologia e Scienze Radiologiche, Sapienza University of Rome, Rome, Italy

Background: Minor salivary gland (MSG) biopsy represents an useful tool not only for the diagnosis of primary Sjogren's Syndrome (pSS) but also to evaluate patients prognosis. Recognition of germinal centers (GCs) by hematoxilin eosin (HE) and/or $\mathrm{IHC}$ staining for follicular dendritic cells (FDC) detection is mandatory, representing a risk factor for lymphoma development. Focus score (FS) is one of the main instrument to quantify MSG impairment, nonetheless quality information regarding the type of infiltrate such as the entity, structure and localization, are lacking.

Objectives: Aim of this study is to find any association of specific histological features of MSG from patients with pSS with the principal clinical and laboratory features. Moreover, to investigate the utility of histological parameters, other than FS or GCs, for characterizing patients.

Methods: Patients with pSS were enrolled in our SS clinic, and clinical/laboratory data (table) referring to the time of MSG biopsy, gathered on a dedicated database. MSG, removed for diagnostic purposes, were preserved as paraffin embedded tissue, then cut and sequentially stained by $\mathrm{H} \& \mathrm{E}$ and IHC [polyclonal rabbit anti-CD3 (lymphocytes T); monoclonal mouse anti-CD20 (lymphocytes B); monoclonal mouse anti-CD21 (FDC)]. Images were collected by Zeiss Axio Scan and analysed (ZEN software) as follows: FS calculation, mean foci area, percentage of infiltration, presence of segregated foci (SF) (specifically, clear evidence of $T$ and $B$ cells area by CD3-CD20 double staining), GCs and lymphoepithelial lesions (LELs) detection.

Results: 53 MSG from patients with pSS were collected and analysed. Patients clinical and laboratory data are reported in table. FS positively correlated with the percentage of infiltration $(p<0.001)$ as well as with the presence of SF $(p=0.005)$, GCs $(p=0.02)$ and LELs $(P=0.005)$. Mean foci area and percentage of infiltration correlated with SF $(p=0.0002$ and $p<0.001$, respectively), GCs $(p=0.0004$ and $p<0.001$, respectively) and LELs (both $p<0.001$ ). SF correlated with GCs and

\begin{tabular}{ll}
\hline Clinical features & Number $\%$ ) \\
Sex (M/F) & $4 / 49$ \\
Mean age at diagnosis (mean士SD, years) & $54.9 \pm 12.1$ \\
Xerophtalmia & $47 / 53(88.6)$ \\
Xerostomia & $47 / 53(88.6)$ \\
Salivary gland swelling & $16 / 53(30.1)$ \\
Arthritis & $8 / 53(15)$ \\
Lymphoma & $0 / 53(0)$ \\
Purpura & $2 / 53(3.7)$ \\
PNS and/or CNS involvement & $1 / 53(1.8)$ \\
Pancreatitis & $1 / 53(1.8)$ \\
Laboratory features & \\
ANA & $39 / 53(73.6)$ \\
Anti-Ro/SSA & $17 / 53(32.1)$ \\
Anti-La/SSB & $14 / 53(26.4)$ \\
Hypergammaglobulinaemia & $18 / 53(33.9)$ \\
Rheumatoidfactor & $10 / 53(19)$ \\
Leukopenia & $8 / 53(15)$ \\
Hypocomplementemia & $6 / 53(11.3)$ \\
Monoclonal component & $8 / 53(15)$ \\
Cryoglobulinaemia & $2 / 53(3.7)$ \\
\hline
\end{tabular}

LEL $(\mathrm{p}<0.001)$. Anti nuclear antibodies $(A N A)$ were associated with the presence of $S F(p=0.029, O R=5.7 \mathrm{Cl}=1.1-28.8)$ while gland swelling was associated with the presence of $\mathrm{GCs}(\mathrm{p}=0.043$, OR=4, $\mathrm{Cl}=1.1-15)$.

Conclusions: The FS was associated with the presence of GCs and LELs, as well as with more organized infiltrates characterized by segregation in $T$ and $\mathrm{B}$ areas (SF), thus representing an useful tool which mirrors the risk of lymphoma. From our study, the qualitative characteristics of the biopsy, including SF, percentage of infiltration or the mean foci area, appear to be strictly linked. Moreover, their association with the presence of GCs and LELs supports the importance to consider also these features during histological examination. The lack of correlation between histological parameters and clinical/laboratory features might reveal a weaker connection between histological findings and specific SS phenotypes except for the relationship between glandular swelling and GCs which confirms how this clinical aspect should be considered as a risk factor for lymphoma development.

Disclosure of Interest: None declared

DOI: 10.1136/annrheumdis-2017-eular.5862

\section{SAT0306 ASSOCIATION BETWEEN QUALITY OF SLEEP, QUALITY OF LIFE AND DISEASE ACTIVITY IN PATIENTS WITH SYSTEMIC LUPUS ERYTHEMATOSUS}

S. Monov $^{1}$, D. Monova ${ }^{2}$, M. Ivanova ${ }^{3} .{ }^{1}$ Department of Rheumatology, Medical University - Sofia; ${ }^{2}$ Department of Internal Diseases, Medical University - Sofia, Medical Institute; ${ }^{3}$ Department of Internal Diseases, Medical Institute, Sofia, Bulgaria

Background: Systemic Lupus Erythematosus (SLE) patients are known to have sleep disturbances. Quality of sleep may affect quality of life, but this association has not been systematically evaluated.

Objectives: The aim of this study was to examine the association of quality of sleep, quality of life and SLE disease activity in patients diagnosed with SLE.

Methods: 132 SLE patients with a confirmed diagnosis of SLE according to the ACR classification criteria were enrolled in this study. The patients completed the following questionnaires: the Pittsburgh Sleep Quality Index (PSQI), the 12 item Short Form Health Survey (SF-12), the Lupus Patient-Reported Outcome tool (LupusPRO), SLE Quality of Life Questionnaire (SLE - QoL). Clinical information, including the SLE Disease Activity Index (SLEDAI), was obtained from medical records. Student's t-test, ANOVA, Pearson correlation measured were used in statistical analysis.

Results: The majority of the participants $(84,4 \%)$ had sleep disturbances (PSQI $>5$ ). Total PSQI score was weakly associated with all of the SF-12 subcategories and showed weak to moderate associations with the LupusPRO subcategories $(r<0,05)$, except for "medication" $(r<0,20)$. "Sleep duration" was not associated with any of the SF-12 or LupusPRO subcategories. "Sleep efficiency" was weakly associated with "physical health", "physical function", and "pain" in the SF-12 and LupusPRO. "Sleep quality" and "sleep disturbances" were weakly associated with "pain" and the "emotional" and "mental" subcategories in the SF-12 and LupusPRO. SLE - QoL was significantly higher in patients with good sleep.

Conclusions: We found that quality of sleep, especially "sleep efficiency", was poor for the majority of patients with SLE. Quality of sleep was associated with various aspects of quality of life, especially pain, vitality, and emotional health. Management of pain and emotional health may be important for improving quality of sleep in SLE patients.

Disclosure of Interest: None declared

DOI: 10.1136/annrheumdis-2017-eular.1450

\section{SAT0307 RECURRENCE RATE OF THROMBOSIS FOR PATIENTS WITH ANTI-PHOSPHOLIPID ANTIBODIES INITIALLY AND DISAPPEARED LATER AFTER THROMBOSIS}

S.M. Kim ${ }^{1}$, J. Kim ${ }^{2}$, Y. Kim ${ }^{3}$, S.W. Kang ${ }^{2}$, S.-C. Shim ${ }^{2}$, S.-J. Yoo ${ }^{3} .{ }^{1}$ Internal Medicine, Chungbuk National University Hospital, Cheong-ju; ${ }^{2}$ Internal Medicine, School of Medicine Chungnam National University; ${ }^{3}$ Internal Medicine, Chungnam National University Hospital, Daejeon, Korea, Republic Of

Background: In case of anti-phospholipid syndrome, anticoagulants are recommended. However, there were no data about recurrence rate for thrombosis in patients with anti-phospholipid antibodies (APS) initially which disappeared later. Objectives: We compared recurrence rate of thrombosis between negative conversion group and control group.

Methods: We reviewed the medical records of patients diagnosed with thrombosis such as cerebral infarct, myocardial infarct, deep vein thrombosis, or thrombosis of other vessels at a tertiary medical center from 2000 to October 2016. Of these, 14 patients whose APA status was converted from positive to negative after more than 12 weeks were enrolled as negative conversion group. Forty-six patients without APA were matched with the ratio of 1:3 1:4 according to age, sex, thrombosis type (arterial or venous) and therapeutic agents as control group. Results: There was no difference between negative conversion group and control group in smoking status, presence of diabetes or hypertension, duration from the thrombosis to last visits or to recurrence, the proportion of patients taking glucocorticoids. There was no difference in the overall recurrence of thrombosis between two groups [negative conversion group, $3 / 14$ (21\%) vs. control group, 